\title{
Trial Disease Milestones Domain
}

National Cancer Institute

\section{Source}

National Cancer Institute. Trial Disease Milestones Domain. NCI Thesaurus. Code

C147270.

A subject domain utilized for the submission of information encompassing and representing data, vocabulary or records related to trial disease milestones. 